\title{
The autoimmune rheumatic diseases - an introduction
}

Ian Giles

Alan Salama

lan Giles PhD FRCP is a Reader in Rheumatology at UCL and Honorary Consultant at University College London Hospitals, UK. He has a specialist interest in antiphospholipid syndrome and pregnancy outcomes in rheumatic disease. Competing interests: none declared.

Alan Salama PhD FRCP is a Professor in Nephrology at Royal Free Hospital, London, UK.

Competing interests: none declared.

The autoimmune rheumatic diseases (ARDs) are a heterogeneous group of disorders characterized by dysregulation of the immune system leading to tissue injury. These conditions present challenges in terms of both diagnosis and management. An increased understanding of pathogenesis, newer diagnostic markers and the availability of targeted biological treatments has, however, improved the outlook for patients. We are delighted to introduce complementary articles written by opinion leaders in these conditions covering the diverse spectrum of ARDs that physicians are likely to encounter in routine practice, with practical guidance on diagnosis and management as well as mention of exciting new developments.

An approach to immunological testing in different clinical circumstances is given by Drs Derrett-Smith and Campochiaro, who provide an overview of the role of autoantibodies in the diagnosis and monitoring of ARDs, and explain what additional supportive diagnostic evidence may be required. It is important to bear in mind that overlap syndromes also exist and variants of the scenarios described occur.

Distinctions in autoantibody profiles between disease subtypes are well described for certain ARDs but increasingly recognized in others. Professor Herrick describes how two subtypes of systemic sclerosis (limited cutaneous, diffuse cutaneous) have very different natural histories and prognoses, but points out that advances in treatment are leading to improved quality of life and increased survival. Drs Clarke and Giles mention that increasing evidence implicates non-thrombotic mechanisms in the pathogenesis of obstetric antiphospholipid syndrome (OAPS) by antiphospholipid antibodies, and that progression to thrombosis in patients with OAPS is scarce compared with thrombotic APS, suggesting that OAPS is a distinct subset within APS.

Interest in new biological therapies targeting various immune pathways remains high, although trials of new agents have not always been successful. In systemic lupus erythematosus, Drs Yeo and Dias and Professor Isenberg detail success with belimumab but also list various other failed agents. In their overview of vasculitides, Drs Kidder, Dospinescu, Karabayas and Basu emphasize the differences and similarities between vasculitides, and highlight the risk-benefits of current treatments using conventional and biological agents. The diagnostic difficulties and management strategies in medium vessel vasculitis are explored by Drs Hewins and 
King[cmw1], while Dr Karangizi and Professor Harper focus on small vessel vasculitis and emphasize that rapid diagnosis and treatment is vital to prevent organ damage. In ANCA-associated vasculitis, rituximab or cyclophosphamide with high-dose corticosteroids is equally effective as induction therapy, providing options for treatment individualization. However, relapses remain common, and maintenance treatment type and duration must also be tailored to the individual. In their overview of large vessel vasculitis, Drs Tracy, Cardy and Carruthers describe an emerging role for biological therapies and increasing use of temporal artery ultrasound in diagnosis.

Alternative therapeutic options are also being investigated. Professor Herrick describes fibrotic and vascular features of systemic sclerosis that can now be targeted, while Drs Parker, Lilleker, Roberts and Chinoy explain that noninflammatory pathways are being scrutinized as future targets for treatment of autoimmune myopathies. Finally, Drs Dumusc and Rao and Professor Bowman remind us that better use of symptomatic therapies can still make a big difference to patients with Sjögren's syndrome, while trials of biological therapy are underway. 